\title{
First-principles GW calculations for fullerenes, porphyrins, phtalocyanine, and other molecules of interest for organic photovoltaic applications
}

\author{
X. Blase, C. Attaccalite, V. Olevano \\ Institut Néel, CNRS and Université Joseph Fourier, \\ B.P. 166, 38042 Grenoble Cedex 09, France, \\ and \\ European Theoretical Spectroscopy Facility (ETSF), Grenoble, France.
} (Dated: May 31, 2018)

\begin{abstract}
We evaluate the performances of ab initio GW calculations for the ionization energies and HOMOLUMO gaps of thirteen gas phase molecules of interest for organic electronic and photovoltaic applications, including the $\mathrm{C}_{60}$ fullerene, pentacene, free-base porphyrins and phtalocyanine, PTCDA, and standard monomers such as thiophene, fluorene, benzothiazole or thiadiazole. Standard $\mathrm{G}_{0} \mathrm{~W}_{0}$ calculations, that is starting from eigenstates obtained with local or semilocal functionals, significantly improve the ionization energy and band gap as compared to density functional theory KohnSham results, but the calculated quasiparticle values remain too small as a result of overscreening. Starting from Hartree-Fock-like eigenvalues provides much better results and is equivalent to performing self-consistency on the eigenvalues, with a resulting accuracy of $2-4 \%$ as compared to experiment. Our calculations are based on an efficient gaussian-basis implementation of GW with explicit treatment of the dynamical screening through contour deformation techniques.
\end{abstract}

PACS numbers: 71.15.-m,71.15.Ap,71.15.Qe,71.20.Rv

\section{INTRODUCTION}

The flexibility in the synthesis of novel molecules and polymers is an important advantage of organic photovoltaics as compared to the inorganic route 1.2 . Despite a rather limited quantum efficiency, the possibility to tailor the solubility, cristallinity and electronic properties of the building molecular units is offering much means to improve on the actual best cells, such as those based on the combination of acceptor fullerene derivatives and derivatives of polythiophene as donors ${ }^{3.4}$. In particular, it has been shown that there are strong correlations between the "band offsets" at the donor/acceptor interface and the open circuit voltage or the driving force for separating the hole and electron of the photoinduced excitons ${ }^{5,6}$. The ability to tune the electronic affinity and ionization energy of the donor and acceptor molecules, under the constraint that sun light absorption should be kept as large as possible, is a current and intense field of research ${ }^{7-10}$. There is therefore much interest in developing efficient quantum simulation methods allowing to provide the spectroscopic and optical properties of standard molecules with both a reasonnable computer cost and accuracy.

For isolated molecules, an excellent trade-off between computer cost and accuracy for the calculations of the ionization energy and electronic affinity can be found with the so-called $\triangle \mathrm{SCF}$ approach using hybrid functionals such as PBE0 and B3LYP obtained by admixture of a fixed amount of Fock exchange $\frac{11,12}{12}$. However, these techniques cannot be used for extended systems such as bulk semiconductors, molecules deposited on a surface or in solution, and the percentage of Fock exchange needed for obtaining good results with these functionals is expected to change from isolated molecules to bulk sys- tems. For the same reasons, the "Kohn-Sham" ionization energies, electronic affinities and band gaps as obtained from the eigenvalues of the Hamiltonian may be certainly improved with hybrid functionals as compared to (semi)local ones, but again the amount of Fock exchange needed to get accurate results may change from one system to another.

A technique based on many-body perturbation theory (MBPT), namely the GW approximation ${ }^{13}-16$, has shown excellent results for the evaluation of the band edges and band gaps of extended bulk systems 17 . Distinct from the perturbative techniques developed by the quantum chemistry community to build up correlations from the Hartree-Fock solution ${ }^{18}$, such an approach is generally derived from functional derivative techniques 13,19 yielding an exact (non-perturbative) set of self-consistent (closed) relations between the one-body Green's function $\mathrm{G}$, the polarizability $P$, the dynamically screened Coulomb potential W, the "exchange and correlation" self-energy $\Sigma$ and the so-called vertex corrections $\Gamma$, which is related to the variation of the self-energy with respect to an external pertubation. In practice, neglect of vertex corrections leads to the so-called "GW" approximation for the self-energy which can be loosely described as a generalization of the Hartree-Fock method by replacing the bare Coulombian with a dynamically screened Coulomb interaction. The ingredients needed to proceed through the GW calculations pave further the way to Bethe-Salpeter calculations $\frac{16}{6}$ aiming at exploring optical absorption spectra as an alternative to timedependent DFT. While decades of expertise exist for appraising the performances of the GW approximation in the case of extended bulk systems, the application of such MBPT approaches to organic molecules in the gas phase, and in particular molecules of interest for photovoltaic 
applications $\underline{20-24}$, remain extremely scarce, a situation that can be mostly attributed to the associated computational cost for molecules such as fullerene derivatives or porphyrins containing several dozens of atoms. As a result, an understanding of the merits of such an approach in the case of organic molecular systems, as compared to well-established quantum chemistry techniques, is still in its infancy.

We present in this work a GW study of the quasiparticle properties of thirteen of the most standard molecules involved in organic electronic and photovoltaic devices, including the $C_{60}$ fullerene, the freebase $21 \mathrm{H}, 23 \mathrm{H}$-porphine $\left(\mathrm{H}_{2} \mathrm{P}\right)$, tetraphenylporphyrin $\left(\mathrm{H}_{2} \mathrm{TPP}\right)$, and phtalocyanine $\left(\mathrm{H}_{2} \mathrm{Pc}\right)$, and the $3,4,9,10-$ perylene tetracarboxylic acid dianydride (PTCDA) (see Fig. 10. We also study anthracene, tetracene, and pentacene, $\pi$-conjugated molecules of interest for organic electronics, even though not as such for optical applications, and for which experimental band gap data are available. Finally, the tiophene, fluorene, benzothiazole, 2,1,3-benzothiadiazole and 1,2,5-thiadiazole monomers, building blocks of common donor polymers, are also investigated 25,26 . Our results suggest that while the standard non-self-consistent $\mathrm{G}_{0} \mathrm{~W}_{0}$ calculations based on Kohn-Sham eigenstates with (semi)local functionals certainly improves on the DFT results, the $\mathrm{G}_{0} \mathrm{~W}_{0}$ ionization energy and HOMO-LUMO gap remain underestimated as compared to experiment. A simple partial selfconsistency on the eigenvalues only, or the use of HartreeFock-like eigenvalues in a one-shot $\mathrm{G}_{0} \mathrm{~W}_{0}$ calculation, allows to obtain much improved results. We show in particular that these simple schemes lead to an average error of $\sim 0.3 \mathrm{eV}$ for the ionization energies and $0.1-0.2 \mathrm{eV}$ for the band gaps.

Our paper is organized as follows. In section (II), we briefly describe our implementation of the GW formalism within a gaussian-basis, including details about the evaluation of the Coulomb matrix elements. In section (III), our results for the ionization energy and HOMO-LUMO gap of selected molecules are presented and compared to existing experimental results. The importance of a simple self-consistency on the eigenvalues is discussed. Section (IV) describes a simplified non-self-consistent approach based on an approximate perturbative HartreeFock starting point for building the Green's function and screened Coulomb potential. We conclude in section (V).

\section{METHODOLOGY}

Our code is based on a gaussian-basis implementation of the GW formalism and builds on a previous implementation of calculating the inverse dielectric matrix using numerical strictly localized orbitals $\$ 27$. To avoid dealing with numerical basis, the present implementation now expands the needed two-point operators (bare and screened Coulomb potentials, susceptibilities, etc.) on an "auxiliary" gaussian basis composed of one-center atomic-like orbitals, with real spherical harmonics for the angular part and a radial dependence composed of gaussian functions. The use of such an auxiliary basis, commonly implemented in several DFT quantum chemistry codes to express the charge density for ground-state or excitedstate $\frac{28}{}$ calculations, allows to greatly speed up the evaluation of e.g. the Coulomb matrix elements. We discuss these points in the following subsections.

\section{A. General formalism}

With the notations of Ref. 29, we introduce for any two-point function $f\left(\mathbf{r}, \mathbf{r}^{\prime}\right)$ the $\langle f>$ and $[f]$ matrices in the auxiliary basis related through:

$$
\begin{aligned}
{[f]_{\mu, \nu} } & =\iint d \mathbf{r} d \mathbf{r}^{\prime} \mu^{*}(\mathbf{r}) f\left(\mathbf{r}, \mathbf{r}^{\prime}\right) \nu\left(\mathbf{r}^{\prime}\right) \\
<f> & =S^{-1}[f] S^{-1} \\
f\left(\mathbf{r}, \mathbf{r}^{\prime}\right) & =\sum_{\mu, \nu} \mu(\mathbf{r})<f>_{\mu, \nu} \nu^{*}\left(\mathbf{r}^{\prime}\right)
\end{aligned}
$$

where $\mu$ and $\nu$ are elements of the basis and $S$ is the overlap matrix. The standard Dyson equation relating the dynamically screened Coulomb potential $W(\omega)$ to the bare Coulomb one $(v)$ can then be written:

$$
<W(\omega)>=<v>+<v>\left[\chi^{0}(\omega)\right]<W(\omega)>
$$

with $\chi^{0}$ the unscreened (free-electron) susceptibility:

$$
\begin{aligned}
{\left[\chi^{0}(\omega)\right]_{\mu, \nu} } & =\sum_{\text {spins }} \sum_{i}^{\text {occ unocc }} \sum_{j}<\phi_{i}|\mu| \phi_{j}><\phi_{j}|\nu| \phi_{i}> \\
& \times\left(\frac{1}{\omega+\varepsilon_{i}-\varepsilon_{j}+i \delta}-\frac{1}{\omega-\varepsilon_{i}+\varepsilon_{j}-i \delta}\right)
\end{aligned}
$$

where $\delta=0^{+}$. The input $\left(\phi_{i}, \varepsilon_{i}\right)$ are one-body eigenstates and related eigenvalues traditionaly taken as the Kohn-Sham solutions of a ground-state DFT calculation. In the present paper, we start with a standard DFT/LDA calculation but as discussed below, this may not constitute the best starting point for molecular systems. The knowledge of the dynamical screened Coulomb potential $W(\omega)$ allows to build the non-local and energy dependent self-energy operator $\Sigma$, which accounts for exchange and correlation in the present quasiparticle formalism ${ }^{13}$ and reads:

$$
\begin{aligned}
\Sigma^{G W}\left(\mathbf{r}, \mathbf{r}^{\prime} \mid \mathbf{E}\right) & =\frac{i}{2 \pi} \int d \omega e^{i 0^{+} \omega} G\left(\mathbf{r}, \mathbf{r}^{\prime} \mid E+\omega\right) W\left(\mathbf{r}, \mathbf{r}^{\prime} \mid \omega\right) \\
G\left(\mathbf{r}, \mathbf{r}^{\prime} \mid \omega\right) & =\sum_{n} \phi_{n}(\mathbf{r}) \phi_{n}^{*}\left(\mathbf{r}^{\prime}\right) /\left(\omega-\varepsilon_{n} \pm i \delta\right)
\end{aligned}
$$






(b)

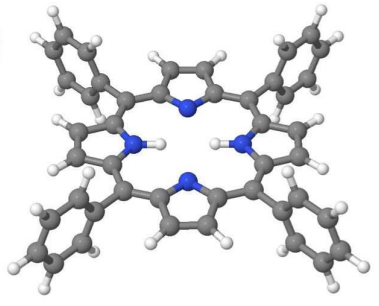

(c)

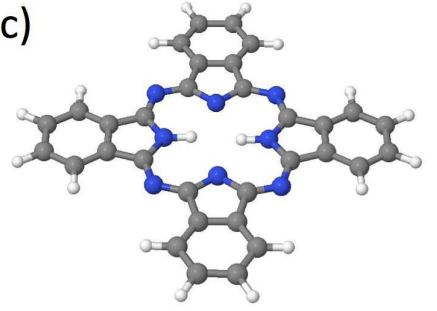

(d)

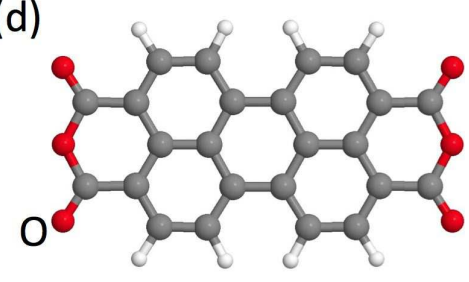

(e)

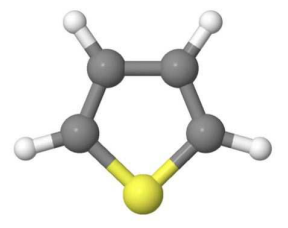

(f)

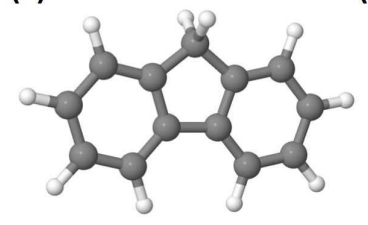

(g)

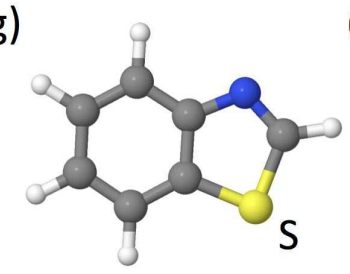

(h)

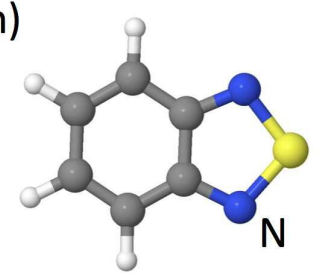

(i)

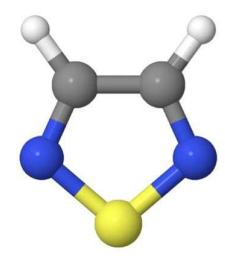

FIG. 1. (Color online) Symbolic representation of (a) 21H,23H-porphine $\left(\mathrm{H}_{2} \mathrm{P}\right)$, (b) tetraphenylporphyrin $\left(\mathrm{H}_{2} \mathrm{TPP}\right)$, (c) phtalocyanine $\left(\mathrm{H}_{2} \mathrm{Pc}\right)$ (d) 3,4,9,10-perylene tetracarboxylic acid dianydride (PTCDA), (e) thiophene, (f) fluorene, (g) benzothiazole, (h) 2,1,3-benzothiadiazole and (i) 1,2,5-thiadiazole. Small white atoms are hydrogen atoms, grey atoms are carbon atoms while red/blue/yellow atoms are oxygen/nitrogen/sulfur atoms respectively.

where the time-ordered Green's function $G$ is again built from the $\left(\phi_{i}, \varepsilon_{i}\right)$ eigenstates. The sign of the $\delta$ infinitesimal insures that the occupied (unoccupied) states correspond to poles in the fourth (second) quadrants. Again, the choice of the "best" input $\left(\phi_{i}, \varepsilon_{i}\right)$ for the building of $\mathrm{G}$ will be discussed below.

This implementation is formally equivalent to that of Ref. 29 except that we go beyond the plasmon-pole model and proceed with the explicit calculation of the frequency integral for the correlation part of the self-energy, $\Sigma_{c}^{G W}=\Sigma^{G W}-\Sigma_{x}$, with $\Sigma_{x}$ the Fock operator. We use contour deformation techniques with an integration along the imaginary axis complemented by the evaluation of the poles in the first and third quadrant for states away from the band edges 15,30 :

$$
\Sigma_{c}^{G W}\left(\mathbf{r}, \mathbf{r}^{\prime} \mid E\right)=\sum_{n} \phi_{n}(\mathbf{r}) \phi_{n}^{*}\left(\mathbf{r}^{\prime}\right) \mathcal{V}_{n}\left(\mathbf{r}, \mathbf{r}^{\prime} \mid E\right)
$$

with, introducing $\tilde{W}=W-v, E_{F}$ the Fermi level, and $\theta$ the Heaviside step function:

$$
\begin{aligned}
\mathcal{V}_{n}\left(\mathbf{r}, \mathbf{r}^{\prime} \mid E\right)= & \tilde{W}\left(\mathbf{r}, \mathbf{r}^{\prime} \mid \varepsilon_{n}-E\right)\left[\theta\left(E-\varepsilon_{n}\right)-\theta\left(E_{F}-\varepsilon_{n}\right)\right] \\
& -\int_{0}^{+\infty} \frac{d \omega}{\pi} \frac{E-\varepsilon_{n}}{\left(E-\varepsilon_{n}\right)^{2}+\omega^{2}} \tilde{W}\left(\mathbf{r}, \mathbf{r}^{\prime} \mid i \omega\right)
\end{aligned}
$$

A change of variable allows to fold the smooth function $\tilde{W}(i \omega)$ onto the finite $[0,1]$ interval where Gaussian quadrature with as little as 12 gaussian points is sufficient to reach convergency. An analytically integrable tail is added/subtracted to avoid instabilities with the integrand for $\omega \rightarrow 0$ when $E \rightarrow \varepsilon_{n}$.
The first order perturbation theory self-energy correction to the DFT Kohn-Sham eigenvalues is extrapolated to the quasiparticle energies by a Taylor expansion, namely:

$$
\varepsilon_{n}^{Q P}=\varepsilon_{n}+Z_{n}<\phi_{n}\left|\Sigma^{G W}\left(\varepsilon_{n}\right)-V_{x c}^{L D A}\right| \phi_{n}>
$$

where $Z_{n}$ is the renormalization factor defined as:

$$
1 / Z_{n}=1-\left[\partial \Sigma^{G W} / \partial E\right]_{\varepsilon=\varepsilon_{n}} .
$$

with $\left(\varepsilon_{n}, \phi_{n}\right)$ the LDA Kohn-Sham eigenvalues and eigenstates in the present case.

\section{B. Gaussian basis}

The auxiliary basis used to expand the two-point functions reads: $\mu(\mathbf{r})=\exp \left(-\alpha r^{2}\right) r^{l} R_{l}^{m}(\hat{r})$ with $R_{l}^{m}(\hat{r})$ the real-spherical harmonics and $(\hat{r})$ the angular components of the r-vector. It is computationally more efficient to work with the $R_{l}^{m}$ instead of the standard $Y_{l}^{m}$ complex harmonics with the following relation:

$$
R_{l}^{m}(\hat{r})= \begin{cases}\left.{ }_{Y_{l}^{m}}^{m}(\hat{r})+(-1)^{m} Y_{l}^{-m}(\hat{r})\right] / \sqrt{2} & (\mathrm{~m}>0) \\ Y_{l}^{m}(\hat{r}) & (\mathrm{m}=0) \\ {\left[Y_{l}^{-m}(\hat{r})-(-1)^{m} Y_{l}^{m}(\hat{r})\right] / \sqrt{2}} & (\mathrm{~m}<0)\end{cases}
$$

The products $r^{l} R_{l}^{m}(\hat{r})$ yield the standard expressions $\left(x, y, z, x y, y z, x^{2}-y^{2}\right.$, etc.) for the $p, d$, etc. orbitals (within constant factors). We briefly recall that the main advantage of a gaussian radial part (as compared to numerical or Slater-type orbitals) is that the product of 
two gaussians centered on atoms $A$ and $B$ with decay coefficients $\alpha_{1}$ and $\alpha_{2}$ yields a gaussian centered on $C=\left(\alpha_{1} A+\alpha_{2} B\right) /\left(\alpha 1+\alpha_{2}\right)$ with a decay constant $\gamma=\alpha_{1} \alpha_{2} /\left(\alpha_{1}+\alpha_{2}\right)$. Further, the $r^{l} R_{l}^{m}(\hat{r})$ can easily be "shifted" from one center to another with for sake of illustration:

$$
\begin{aligned}
\left(x-x_{A}\right)\left(y-y_{A}\right) & =\left(x-x_{C}\right)\left(y-y_{C}\right) \\
& +\left(y_{C}-y_{A}\right)\left(x-x_{C}\right) \\
& +\left(x_{C}-x_{A}\right)\left(y-y_{C}\right)+\text { constant }
\end{aligned}
$$

showing that a $d_{x y}$ orbital centered on $A$ can be easily expressed as a function of $(s, p)$ and $d_{x y}$ orbitals centered on $C$. Such trivial expressions allow to express multicenter overlaps in terms of one-center integrals.

In the present work, our calculations start with a DFT calculation of the structural and electronic properties of the molecules of interest using the Siesta package 31 . We use a "double- $\zeta+$ polarization" (DZP) basis"32 and standard norm-conserving pseudopotentials. Since the Siesta package uses "numerical" orbitals, we first fit the numerical radial part by up to five contracted gaussians 34 in order to exploit the relations briefly sketched above. As such, both the "ground-state" DFT basis and the auxiliary basis are based on gaussians. Beyond the analycity of the gaussian basis, our choice was also motivated by the possibility of using eigenstates generated by standard chemistry codes with all electron approaches and/or hybrid functionals, providing for some systems possibly a better starting point for MBPT calculations (see discussion below). We labeled our code "Fiesta" as an attempt to extend the "Siesta" package to excited state properties.

Contrary to the planewave case, the auxiliary basis for the two-point response functions is larger than the ground-state basis. Following Kaczmarski and $\operatorname{coworkers}^{35}$, we typically adopt for first raw elements such as carbon, nitrogen and oxygen, $4 s, p, d$ sets of gaussian orbitals, that is 36 orbitals per atom, while $3 s, p, d$ gaussian orbitals are sufficient for hydrogen. We show below that such a basis is large enough for the studied organic systems. In the case of sulfur, $f$-channel orbitals are added. With such a basis, a typical $\mathrm{G}_{0} \mathrm{~W}_{0}$ calculation with full dynamics for our largest molecule $\left(\mathrm{H}_{2} \mathrm{TPP}\right)$ can be performed within one day on a single standard processor. Better timings and scaling may be obtained upon implementing the recently introduced techniques allowing to avoid summation over the conduction states $23,36-38$, or techniques decoupling the sum over valence and conduction states 39 , even though the number of unoccupied states is rather limited with standard DZP or larger TZDP basis.

The choice of the "optimal" $\alpha$-coefficients, controlling the localization of the basis orbitals, is a difficult question. Auxiliary basis have been implemented in many quantum chemistry codes in order to fit the charge density and speed up the calculation of the Coulomb inte-

\begin{tabular}{|l|c|c|c|c|c|c|}
\hline auxiliary basis & \multicolumn{2}{|c|}{ anthracene } & \multicolumn{2}{|c|}{$\mathrm{H}_{2} \mathrm{P}$} & \multicolumn{2}{c|}{$\mathrm{C}_{60}$} \\
\hline $\mathrm{ng}$ in $\alpha_{\min } \rightarrow \alpha_{\max }$ & IE & gap & IE & gap & IE & gap \\
\hline 3 in $0.2 \rightarrow 3.2$ & 6.83 & 6.02 & 6.49 & 4.67 & 7.21 & 4.08 \\
\hline 4 in $0.2 \rightarrow 3.2$ & 6.89 & 6.15 & 6.56 & 4.79 & 7.29 & 4.44 \\
\hline 5 in $0.2 \rightarrow 3.2$ & 6.86 & 6.14 & 6.52 & 4.76 & 7.30 & 4.37 \\
\hline 4 in $0.15 \rightarrow 3.2$ & 6.89 & 6.15 & 6.52 & 4.74 & 7.40 & 4.47 \\
\hline 5 in $0.15 \rightarrow 3.2$ & 6.82 & 6.06 & 6.56 & 4.77 & 7.29 & 4.36 \\
\hline 5 in $0.15 \rightarrow 3.5$ & 6.83 & 6.08 & 6.51 & 4.75 & 7.28 & 4.33 \\
\hline
\end{tabular}

TABLE I. Evolution of the ionization (IE) and band gap energies of selected molecules as a function of the carbon auxiliary basis, changing the number (ng) of gaussians per l-channel, the $\alpha_{\min }$ and $\alpha_{\max }$ coefficients. Results are in $\mathrm{eVs}$.

grals. The coefficients of the charge density on the auxiliary basis are optimized using "identity rules" $\underline{\underline{4}}$ but not the decay coefficients in the exponentials. Years of expertise in the quantum chemistry community yielded reliable auxiliary basis for the periodic table and numerous tests have shown that high precision can be obtained with such basis provided that they be sufficiently large.

Since the auxiliary basis must project onto products of Kohn-Sham orbitals, optimized basis for all-electron calculations cannot be straightforwardly used for GW calculations starting from ground-state calculations with pseudopotentials. The same guiding lines can however be followed. We adopt in particular the idea of a "tempered" basis 1 -43 a chain of $\alpha$ parameters such that: $\alpha_{i+1} / \alpha_{i}=$ constant, rather than spreading them uniformly between $\alpha_{\min }$ and $\alpha_{\max }$. Such a scheme hinges on the facts that the overlap of two gaussian orbitals is a function of their alpha coefficient ratio and that maintaining a constant overlap between "adjacent" gaussians allows to better span the associated Hilbert space $\frac{43}{2}$. As such, the $\alpha_{\min }, \alpha_{\max }$ and number of gaussian per l-channel being chosen, the other gaussian coefficients are automatically generated.

We adopt the basis proposed by Kaczmarski and coworkers ${ }^{35}$, that is namely gaussians with localization parameters of $(0.2,0.5,1.25,3.2)$ a.u. for the $(s, p, d)$ channels of $\mathrm{C}, \mathrm{O}$, and $\mathrm{N}$ atoms, and gaussians with $\alpha=(0.1,0.4,1.5)$ a.u. for hydrogen. As shown in Table I in the case of anthracene, $\mathrm{H}_{2} \mathrm{P}$ porphyrin and $\mathrm{C}_{60}$, changing the $\alpha_{\min }$ and $\alpha_{\max }$ values, or increasing the number of gaussians in the basis, does not change significantly the results. The case of $C_{60}$ shows however that reducing the number of gaussians to 3 per l-channel yields a significant error on the band gap. We will show below that the results obtained with the present implementation compares rather well with previous available calculations based on another gaussian basis, planewaves (PWs) or combination of gaussians, PWs and Wannier functions.

We conclude this section related to the auxiliary basis by mentioning an important numerical aspect related to the overcompletness of the generated non-orthogonal gaussian basis. While the basis on a given atom can be easily orthogonalized using e.g. a Gram-Schmidt procedure, the overlap between the most diffuse orbitals on ad- 
jacent atoms tend to be also rather large yielding an overlap S matrix "nearly singular". Following the strategy developed in the case of product-basis ${ }^{39.44}$, we rotate our basis to that of the eigenvectors of the overlap S-matrix from which we remove the eigenvectors with eigenvalue smaller than typically $10^{-5}$. In the present case of auxiliary basis, such a truncation does not reduce significantly the size of the basis, but avoid the potential numerical instability associated with inverting the nearly-singular S-matrix and the amplification of errors associated with the $\langle v\rangle=S^{-1}[v] S^{-1}$ transformation (see above). The cost of rotating the Coulomb and $<\phi_{i}|\beta| \phi_{j}>$ matrix elements from the original one-center auxiliary basis $(\beta)$ to the (filtered) S-eigenvectors basis scales as $N^{3}$ and represents a marginal part of the CPU time.

\section{Coulomb matrix elements}

An important ingredient is the evaluation of the Coulomb matrix elements between two auxiliary basis orbitals localized on two different atoms. Exploiting the properties of the Fourier transform (FT) of gaussianbased orbitals, namely:

$$
F T\left[e^{-\alpha r^{2}} r^{l} R_{l}^{m}(\hat{r})\right]=C e^{-\gamma q^{2}} q^{l} R_{l}^{m}(\hat{q})
$$

with $\gamma=1 / 4 \alpha$ and $(\hat{r}, \hat{q})$ the angular components of the $(\mathbf{r}, \mathbf{q})$-vectors in direct and reciprocal space respectively ( $\mathrm{C}$ is a constant), the Coulomb matrix elements reduce to a sum of terms built from the product of one-center overlaps of three real-spherical harmonics $<R_{l}^{m} R_{l^{\prime}}^{m^{\prime}} \mid R_{L}^{M}>$ (related to Gaunt coefficients with $\left|l-l^{\prime}\right| \leq L \leq\left(l+l^{\prime}\right)$ ) times radial integrals $\mathrm{I}\left(\mathrm{l}, \mathrm{l}^{\prime} ; \mathrm{L}\right)$ of the form:

$$
I\left(l, l^{\prime} ; L\right)=\int_{0}^{\infty} d q e^{-\zeta q^{2}} q^{\mu} J_{\nu}\left(-\beta q^{2}\right)
$$

The $<R_{l}^{m} R_{l^{\prime}}^{m^{\prime}} \mid R_{L}^{M}>$ factors are pretabulated. The oscillatory behavior of the Bessel function of the first kind $J_{\nu}$ makes the direct numerical evaluation rather unstable. We prefer to notice that $I\left(l, l^{\prime} ; L\right)$ is straighforwardly related to the ${ }_{1} F_{1}$ confluent hypergeometric functions $\frac{45}{4}$ which, for the needed $\left(l, l^{\prime}\right)$ values, can be expressed in terms of simple functions such as the error function (erf) or the Dawson integral: $\mathrm{F}(\mathrm{z})=\sqrt{\pi} \exp \left(-\mathrm{z}^{2}\right) \operatorname{erfi}(\mathrm{z}) / 2$, with $\operatorname{erfi}(\mathrm{z})=\operatorname{erf}(\mathrm{iz}) / \mathrm{i}$, for which rapidly convergent serial expressions exist $\underline{46}$. This is an important advantage of the auxiliary basis approach that the evaluation of the off-site Coulomb matrix elements is not a costly part of the present GW implementation.

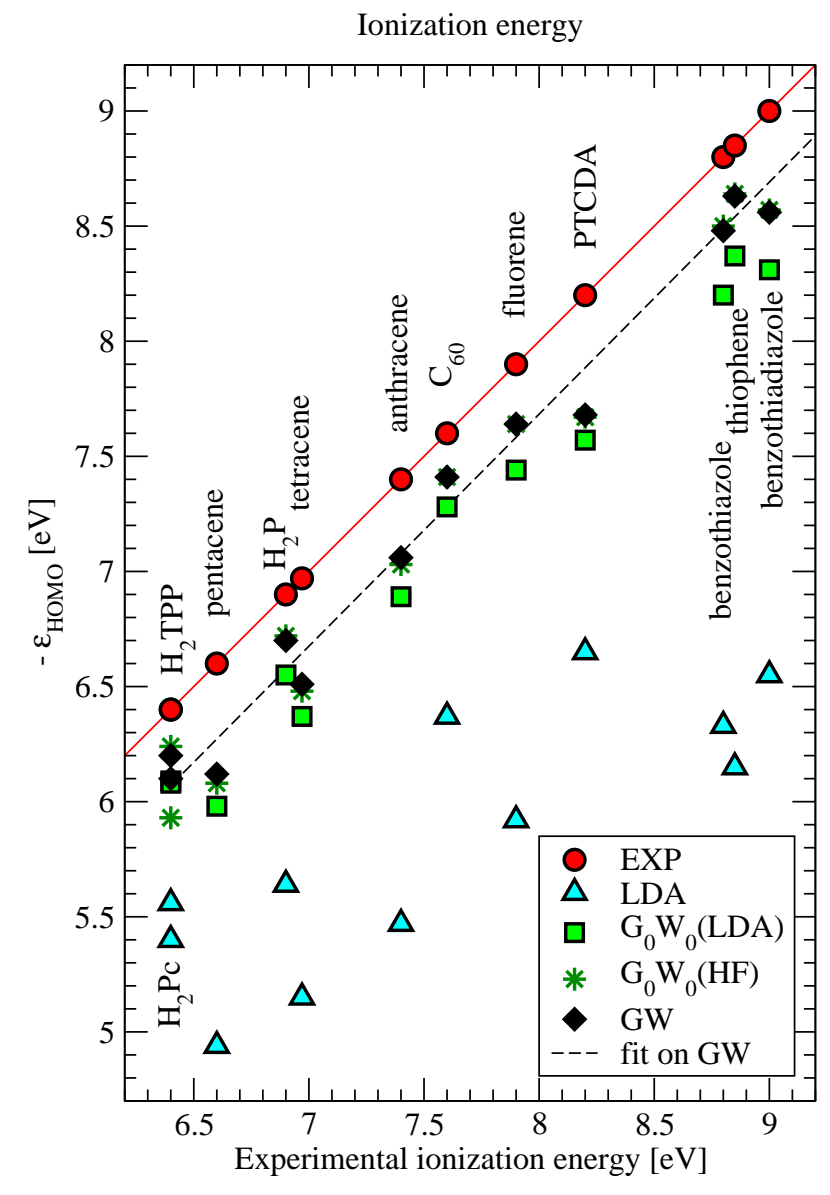

FIG. 2. (Color online) Experimental and theoretical ionization energies in electronvolts. Red circles: experimental values; light blue triangles up: LDA Kohn-Sham HOMO energy; green squares: non-self-consistent $\mathrm{G}_{0} \mathrm{~W}_{0}(\mathrm{LDA})$ value; black diamonds: GW value with self-consistency on the eigenvalues; green stars: non self-consistent $\mathrm{G}_{0} \mathrm{~W}_{0}\left(\mathrm{HF}_{\text {diag }}\right)$ (see text). The black dashed line is a least-square fit of the GW results. The figure has been formatted so as to preserve the same physical scale on both axis.

\section{RESULTS}

\section{A. Ionization energies}

We start by exploring the ionization energy of our selected molecules. While experimental data for the electronic affinity of molecules in the gas phase are scarce, accurate measured ionization energies are much more common 47 . Experimental ionization energies are represented by red circles in Fig. 2 and are given in the last column of Table II. The DFT-LDA ionization energies, as obtained from the opposite sign of the Kohn-Sham highest occupied (HOMO) energy level, are clearly much too small, with an average error of $1.83 \mathrm{eV}$ or $23 \%$ (see blue triangles in Fig. (2). Very similar results are obtained using the HOMO energy value as obtained with a semilocal 


\begin{tabular}{||l|l|l|l|l|l||}
\hline \multicolumn{9}{|c|}{ Ionization energy } \\
\hline LDA-KS & $\mathrm{G}_{0} \mathrm{~W}_{0}(\mathrm{LDA})$ & $\mathrm{GW}$ & $\mathrm{G}_{0} \mathrm{~W}_{0}\left(\mathrm{HF}_{\text {diag }}\right)$ & Experiment \\
\hline tetracene & 5.47 & 6.89 & 7.06 & 7.03 & $7.4^{a}$ \\
pentacene & 5.15 & 6.37 & 6.51 & 6.48 & $6.97^{a}$ \\
$\mathrm{C}_{60}$ & 4.94 & 5.98 & 6.12 & 6.08 & $6.6^{a}$ \\
$\mathrm{PTCDA}$ & 6.37 & 7.28 & 7.41 & 7.41 & $7.6^{a}$ \\
$\mathrm{H}_{2} \mathrm{P}$ & 6.65 & 7.57 & 7.68 & 7.67 & $8.2^{b}$ \\
$\mathrm{H}_{2} \mathrm{TPP}$ & 5.64 & 6.55 & 6.70 & 6.72 & $6.9^{a}$ \\
$\mathrm{H}_{2} \mathrm{Pc}$ & 5.40 & 6.09 & 6.20 & 6.24 & $6.4^{a}$ \\
thiophene & 5.56 & 6.08 & 6.10 & 5.93 & $6.4^{c}$ \\
fluorene & 6.15 & 8.37 & 8.63 & 8.64 & $8.8^{a}$ \\
benzothiazole & 5.92 & 7.44 & 7.64 & 7.64 & $7.9^{a}$ \\
thiadiazole & 6.33 & 8.20 & 8.48 & 8.50 & $8.8^{a}$ \\
benzothiadiazole & 6.22 & 9.65 & 9.89 & 9.90 & $10.1^{d}$ \\
\hline MAE & 6.55 & 8.31 & 8.56 & 8.57 & $9.0^{a}$ \\
\hline
\end{tabular}

TABLE II. Ionization energies in $\mathrm{eV}$ as obtained from the Kohn-Sham eigenvalues (LDA-KS), from non-self-consistent $\mathrm{G}_{0} \mathrm{~W}_{0}(\mathrm{LDA})$ calculations, from a GW calculation with self-consistency on the eigenvalues (GW), and from a non-self-consistent $\mathrm{G}_{0} \mathrm{~W}_{0}$ calculation starting from Hartree-Fock-like eigenvalues $\left(\mathrm{G}_{0} \mathrm{~W}_{0}\left(\mathrm{HF}_{\mathrm{diag}}\right)\right.$, see text). MAE is the average mean error in eV. The average error in percent as compared to the experiment is indicated in parenthesis. ${ }^{a}$ Ref. 47. ${ }^{b}$ Ref. 20. ${ }^{c}$ Ref. 48. ${ }^{d}$ Ref. 49.

functional such as $\mathrm{PBE}^{50}$.

We now turn to $\mathrm{G}_{0} \mathrm{~W}_{0}(\mathrm{LDA})$ calculations, that is nonself-consistent calculations with the Green's function and screened Coulomb potential directly built from the LDA Kohn-Sham eigenstates and eigenvalues. The analysis of the results (column 3 Table I and green squares in Fig. 2) shows that the ionization energies are greatly improved, with an average error of $0.47 \mathrm{eV}$, that is a much reduced $6 \%$ error.

Even though in much better agreement with experiment than LDA or PBE, the discrepancies are still sizeable. As shown below, part of the problem originates in that the "starting" LDA HOMO-LUMO gap is dramatically too small for isolated molecules, inducing a large overscreening. To avoid using some arbitrary scissor operator to open the HOMO-LUMO gap in calculating the susceptibility, we rather perform a restricted selfconsistency by reinjecting the corrected eigenvalues in $\mathrm{G}$ and $\mathrm{W}$ up to convergency. As a matter of fact, no more than three or four iterations are needed to reach convergency within $0.01 \mathrm{eV}$. Such an approximation is labeled GW in the following. This is not a full self-consistent approach as the eigenstates are not updated, with the advantage that the computational cost keeps reasonnable. Full self-consistency without vertex corrections is still debated and seems to yield for small molecular systems results that are not as good as $\mathrm{G}_{0} \mathrm{~W}_{0}$ non self-consistent runs 33 .

The analysis of the results (fourth column Table II and black diamonds in Fig. (2) clearly shows that the selfconsistency on the eigenvalues improves the results for the ionization energy, reducing the average error from $0.47 \mathrm{eV}(6 \%)$ to $0.30 \mathrm{eV}$ (or 3.8\%). Such a discrepancy is still sizeable but much better than the one obtained from the LDA Kohn-Sham HOMO energy. An interesting observation is that the final GW ionization energies gather much closer to a straight line (dotted black line on Fig. (2) parallel to the first diagonal (red "experimental" line) than the LDA data which are much more spread. On a pragmatical point of view, this means that the band offset between two molecules will strongly benefit from cancellation of errors in GW as compared to LDA. In particular, the remaining error $(\sim 0.2 \mathrm{eV})$ on the ionization energy for $\mathrm{C}_{60}$, the most standard acceptor, is nearly identical to the error on the ionization energies of porphyrins and phtalocyanines, which are commonly used donors. We now show that self-consistency, even though limited to updating the eigenvalues only, has an even larger effect on the magnitude of the HOMO-LUMO gaps.

\section{B. HOMO-LUMO gaps}

Due to the lack of experimental values for the electronic affinity, experimental quasiparticle HOMO-LUMO gaps (red circles in Fig. 3) are scarce so that we plot our results as a function of our "best" calculated HOMOLUMO gaps, namely the GW ones. In the case of $\mathrm{C}_{60}$, anthracene, tetracene, and pentacene for which experimental data are available, we observe as expected that the LDA HOMO-LUMO gap (blue triangles) is too small. This is well known in the case of bulk semiconductors but here the discrepancy is much larger, with an average error of $\sim 4.1 \mathrm{eV}$ or $71 \%$.

The $\mathrm{G}_{0} \mathrm{~W}_{0}$ (LDA) HOMO-LUMO gaps (green squares) significantly improves with respect to LDA. Comparing to available $\mathrm{G}_{0} \mathrm{~W}_{0}(\mathrm{LDA})$ data for this class of aromatic molecules, our calculated 6.15 eV HOMO-LUMO gap for anthracene compares well with the $5.97 \mathrm{eV}$ values of Niehaus and coworkers, despite the differences in basis and the treatment of dynamical effects $\$ 1$. Our 
$\mathrm{G}_{0} \mathrm{~W}_{0}(\mathrm{LDA}) 4.79 \mathrm{eV}$ and $4.23 \mathrm{eV}$ HOMO-LUMO gaps for the $\mathrm{H}_{2} \mathrm{P}$ and $\mathrm{H}_{2}$ TPP free-base porphyrins respectively compare further well with the $5 \mathrm{eV}$ and $4.39 \mathrm{eV}$ planewave results of Palummo and coworkers 22 . Similarly, our $\mathrm{G}_{0} \mathrm{~W}_{0}(\mathrm{LDA}) 4.44 \mathrm{eV}$ band gap for $C_{60}$ is in good agreement with the real-space grid formulation of Tiago and coworkers $\frac{21}{2}$ yielding a band gap of $4.36 \mathrm{eV}$. Such comparisons certainly underline the reliability of the present gaussian-basis implementation. Our 4.53 $\mathrm{eV}$ band gap for PTCDA is however smaller than the $4.9 \mathrm{eV}$ band gap found with a previous planewave $\mathrm{GW}$ calculation 20,56,59.

Overall, we remark a systematic underestimation of the $\mathrm{G}_{0} \mathrm{~W}_{0}(\mathrm{LDA}) \mathrm{HOMO}-\mathrm{LUMO}$ gap with respect to the experiment, with an average error for our test molecules of $\sim 0.75 \mathrm{eV}$ or $13 \%$. This contrasts with the case of bulk systems for which the results of $\mathrm{G}_{0} \mathrm{~W}_{0}(\mathrm{LDA})$ are generally in much better agreement with experimental values. Such a behavior can be analyzed by noticing that building the polarizabilities and screened Coulomb potential with LDA eigenvalues, that is in particular with dramatically too small HOMO-LUMO gaps, leads to a significant overscreening. This induces too large a correlation correction " $\mathrm{G}\left(\mathrm{W}-\mathrm{V}^{C}\right)$ " to the Hartree-Fock HOMO-LUMO gap, that is too small a HOMO-LUMO gap.

Even though much better than the Kohn-Sham HOMO-LUMO gap obtained with e.g. the B3LYP functional ${ }^{52}$ (see empty down triangles in Fig. 3), it is desirable to improve the results. Following the simple scheme introduced above, performing self-consistency on the eigenvalues in $\mathrm{G}$ and $\mathrm{W}$, the GW HOMO-LUMO gap is further increased to reach much better agreement with experiment. The MAE is now reduced to $0.22 \mathrm{eV}$ or $3.8 \%$ for our four test molecules. In the case of $C_{60}$, which is the most standard acceptor in organic photovoltaic cells, the excellent agreement with experiment for the band gap value is rather satisfactory. It is interesting to note further that the MAE of $0.22 \mathrm{eV}$ for HOMO-LUMO gaps is close to the $0.3 \mathrm{eV}$ MAE obtained for the ionization energies, suggesting that the electronic affinity is quite well reproduced on the average.

\section{A SIMPLE NON-SELF-CONSISTENT $\mathbf{G}_{0} \mathbf{W}_{0}$ APPROACH BASED ON HARTREE-FOCK-LIKE EIGENVALUES.}

We conclude this study by exploring a simple nonself-consistent $\mathrm{G}_{0} \mathrm{~W}_{0}$ scheme starting from an "ansatz" Hartree-Fock (HF) calculation obtained by removing the exchange-correlation contribution to the LDA eigenvalues and adding the diagonal part of the exchange operator in the LDA basis, namely:

$$
\epsilon_{n}^{" H F "}=\epsilon_{n}^{L D A}+<\phi_{n}^{L D A}\left|\Sigma_{x}-V_{x c}^{L D A}\right| \phi_{n}^{L D A}>,
$$

where $\Sigma_{x}$ and $V_{x c}^{L D A}$ are the Fock and (semi)local exchange-correlation operators. We label this very simple

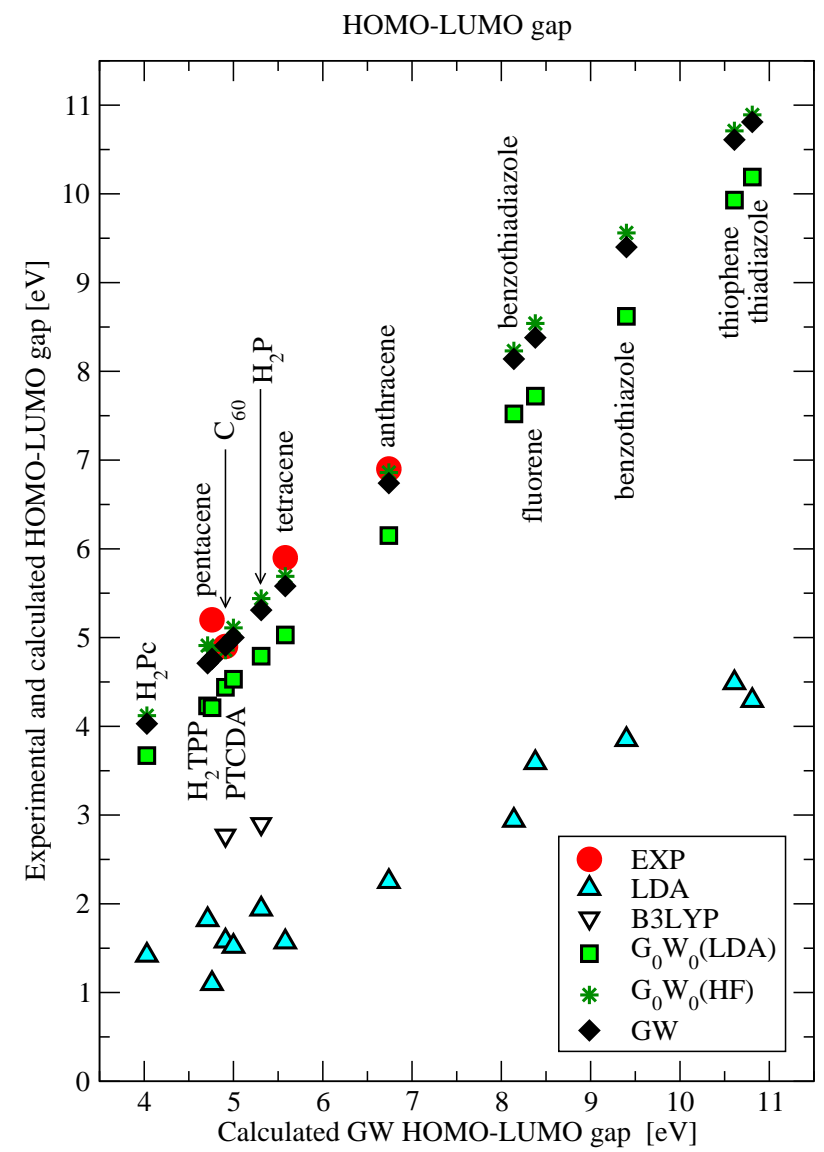

FIG. 3. (Color online) Experimental and theoretical HOMOLUMO gaps in electronvolts. Red circles: experimental values; light blue triangles up: LDA Kohn-Sham HOMO-LUMO gap; green squares: non-self-consistent $\mathrm{G}_{0} \mathrm{~W}_{0}(\mathrm{LDA})$ value; black diamonds: GW value with self-consistency on the eigenvalues; green stars: non-self-consistent $\mathrm{G}_{0} \mathrm{~W}_{0}\left(\mathrm{HF}_{\text {diag }}\right)$ values (see text). The two down-pointing empty triangles are B3LYP/6-31G(d) HOMO-LUMO gap values from Refs. 5255 for $C_{60}$ and the $\mathrm{H}_{2} \mathrm{P}$ porphin.

scheme $\mathrm{G}_{0} \mathrm{~W}_{0}\left(\mathrm{HF}_{\mathrm{diag}}\right)$. This approximation was tested by Hahn, Schmidt and Bechstedt $\underline{60}$ in the case of three small molecules (silane, disilane, water), arguing as we do that the Kohn-Sham eigenvalues are too bad a starting point to evaluate the time-ordered Green's function and the screened potential. Such an approach is also a variation on the $\mathrm{G}_{0} \mathrm{~W}_{0}(\mathrm{HF})$ scheme recently introduced in Ref. 33 which was shown to yield the best ionization energies for small molecules. With increasing size and number of electrons, the part of correlations in the selfenergy is expected to become more important and using Hartree-Fock eigenstates/eigenvalues as a starting point for the much larger systems we study may, in principle, not be better than using (semi)local functionals for generating the starting eigenstates. This is what we now explore.

For sake of comparison, we have studied the two 


\begin{tabular}{|c|c|c|c|c|c|}
\hline \multicolumn{6}{|c|}{ HOMO-LUMO gap } \\
\hline & LDA-KS & $\mathrm{G}_{0} \mathrm{~W}_{0}(\mathrm{LDA})$ & GW & $\mathrm{G}_{0} \mathrm{~W}_{0}\left(\mathrm{HF}_{\mathrm{diag}}\right)$ & Experiment \\
\hline anthracene & 2.25 & 6.15 & 6.74 & 6.86 & $6.9^{a}$ \\
\hline tetracene & 1.57 & 5.03 & 5.58 & 5.69 & $5.9^{a}$ \\
\hline pentacene & 1.10 & 4.21 & 4.76 & 4.86 & $5.2^{a}$ \\
\hline $\mathrm{C}_{60}$ & 1.58 & 4.44 & 4.91 & 5.08 & $4.9^{a}$ \\
\hline MAE & $4.10(71 \%)$ & $0.76(13 \%)$ & $0.22(3.8 \%)$ & $0.10(2 \%)$ & \\
\hline$\overline{\text { PTCDA }}$ & 1.52 & 4.53 & 5.0 & 5.11 & \\
\hline $\mathrm{H}_{2} \mathrm{P}$ & 1.94 & 4.79 & 5.31 & 5.44 & \\
\hline $\mathrm{H}_{2} \mathrm{TPP}$ & 1.82 & 4.23 & 4.71 & 4.91 & \\
\hline $\mathrm{H}_{2} \mathrm{Pc}$ & 1.42 & 3.67 & 4.03 & 4.12 & \\
\hline thiophene & 4.49 & 9.93 & 10.61 & 10.71 & \\
\hline fluorene & 3.59 & 7.72 & 8.38 & 8.54 & \\
\hline benzothiazole & 3.85 & 8.62 & 9.40 & 9.56 & \\
\hline thiadiazole & 4.29 & 10.19 & 10.81 & 10.89 & \\
\hline benzothiadiazole & 2.94 & 7.52 & 8.14 & 8.23 & \\
\hline
\end{tabular}

TABLE III. HOMO-LUMO gap in eV as obtained from the Kohn-Sham eigenvalues (LDA-KS), non-self-consistent $\mathrm{G}_{0} \mathrm{~W}_{0}(\mathrm{LDA}$ ) calculations, a GW calculation with self-consistency on the eigenvalues $(\mathrm{GW})$, and a non-self-consistent $\mathrm{G}_{0} \mathrm{~W}_{0}$ calculation starting from Hartree-Fock-like eigenvalues $\left(\mathrm{G}_{0} \mathrm{~W}_{0}\left(\mathrm{HF}_{\text {diag }}\right)\right.$, see text). MAE is the average mean error in eV for the anthracene, tetracene, pentacene and $\mathrm{C}_{60}$ cases for which experimental band gap data are available. The average error in percent as compared to the experiment is indicated in parenthesis. ${ }^{a}$ Ref. 47 .

small carbon-based conjugated molecules $\mathrm{C}_{2} \mathrm{H}_{2}$ and $\mathrm{C}_{2} \mathrm{H}_{4}$ which were investigated by Rostgaard and coworkers within their full $G_{0} W_{0}(H F)$ scheme. The present $\mathrm{G}_{0} \mathrm{~W}_{0}\left(\mathrm{HF}_{\text {diag }}\right)$ treatment increases the ionization energy by $3.48 \mathrm{eV}$ and $3.80 \mathrm{eV}$ for $\mathrm{C}_{2} \mathrm{H}_{4}$ and $\mathrm{C}_{2} \mathrm{H}_{2}$ respectively as compared to the LDA values. Such corrections compare well with the $3.61 \mathrm{eV}$ and $3.90 \mathrm{eV}$ values obtained within the full $G_{0} W_{0}(H F)$ scheme of Rostgaard and coworkers (as compared to DFT/PBE), emphasizing the reliability of the present simplified approximation.

As compiled in Table II and III (column 5) and in Figs. 2 and 3 (green stars), we do find as well that a single shot $\mathrm{G}_{0} \mathrm{~W}_{0}\left(\mathrm{HF}_{\text {diag }}\right)$ calculation provides results which are in good agreement with the full $\mathrm{GW}$ calculations with self-consistency on the eigenvalues. In particular, the $\mathrm{G}_{0} \mathrm{~W}_{0}\left(\mathrm{HF}_{\text {diag }}\right)$ calculations yield much better results than the $\mathrm{G}_{0} \mathrm{~W}_{0}(\mathrm{LDA})$ scheme. Such a conclusion agrees with that of Rostgaard and coworkers concluding that for small isolated molecules, the full $\mathrm{G}_{0} \mathrm{~W}_{0}(\mathrm{HF})$ scheme actually outperforms a full self-consistent GW calculation where both eigenstates and eigenvalues are updated ${ }^{33}$.

Within the present $\mathrm{G}_{0} \mathrm{~W}_{0}\left(\mathrm{HF}_{\text {diag }}\right)$ approach, the MAE on the ionization energies as compared to experiment is $0.31 \mathrm{eV}$, in good agreement with the $0.4 \mathrm{eV}$ result of Ref. 33 for small molecules. Such an agreement indicates that the present $\mathrm{G}_{0} \mathrm{~W}_{0}\left(\mathrm{HF}_{\text {diag }}\right)$ implementation captures most of the features of a full $G_{0} W_{0}(H F)$ approach, suggesting that LDA and HF eigenfunctions may not too different for this set of molecules, a conclusion oftened discussed in the literature. Further, the error on the band gap, averaged on the calculated values for anthracene, tetracene, pentacene and $\mathrm{C}_{60}$, for which precise experimental data are available, is found to be as small as 0.1 $\mathrm{eV}$ (2\% error). Such values compare very well with accurate quantum chemistry calculations with a scheme, the GW formalism, which can be applied both to finite size and extended systems, and allows to obtain not only the band edges, or frontier orbitals, but also the full quasiparticle spectrum (see note 59).

\section{CONCLUSIONS}

We have explored the performances of several GW approximations for the calculation of the ionization energy and HOMO-LUMO gap of thirteen "large" molecules of interest for photovoltaic applications, including $C_{60}$, freebase porphyrins and phtalocyanine, PTCDA and standard donor monomers such as thiophene. Our calculations are based on a gaussian-basis implementation with full dynamical effects through contour deformation techniques. Due to the dramatic error on the HOMO-LUMO gaps obtained with (semi)local functionals, we find that the standard non-selfconsistent $\mathrm{G}_{0} \mathrm{~W}_{0}$ calculations based on input LDA eigenstates performs rather poorly, in particular in evaluating the HOMO-LUMO gaps. A simple self-consistency on the eigenvalues used to build $\mathrm{G}$ and $\mathrm{W}$ provides much better results. As an even simpler scheme, a non-self-consistent $\mathrm{G}_{0} \mathrm{~W}_{0}\left(\mathrm{HF}_{\text {diag }}\right)$ starting from Hartre-Fock like eigenvalues provides equivalent results. Both the $\mathrm{GW}$ and $\mathrm{G}_{0} \mathrm{~W}_{0}\left(\mathrm{HF}_{\text {diag }}\right)$ approaches provide ionization energies with a mean average error within $\sim 0.3 \mathrm{eV}(\sim 4 \%)$ of the experiment. Concerning the HOMO-LUMO gaps, with a limited number of experimental data, the same $\mathrm{GW}$ and $\mathrm{G}_{0} \mathrm{~W}_{0}\left(\mathrm{HF}_{\text {diag }}\right)$ approaches yield a mean average error of 0.1-0.2 eV (2-4\%), in much better agreement than the $4.1 \mathrm{eV}(71 \%)$ error within DFT/LDA, but also in significantly better agreement than the $0.76 \mathrm{eV}(13 \%)$ error within the "standard" $\mathrm{G}_{0} \mathrm{~W}_{0}(\mathrm{LDA})$ approach. The possibility of performing GW calculations for molecules comprizing several dozens of atoms with reasonnable computer time and accuracy, 
with a scheme allowing to obtain the full quasiparticle spectrum of both finite size and extended systems, opens the way to the investigation of organic photovoltaic systems with techniques that may possibly compete with well-established quantum chemical approaches.

Acknowledgements - X.B. is indebted to Marc Cassida for suggesting numerous references, Pascal Quemerais for pointing out the relations between the radial integrals involved in the Coulomb matrix elements evaluation and the ${ }_{1} F_{1}$ confluent hypergeometric functions, Julian Gale for discussions on the gaussians fit of the radial part of numerical orbitals, and Brice Arnaud for suggesting techniques to stabilize the imaginary axis integration in the contour deformation approach. Calculations have been performed on the CIMENT plateform in Grenoble thanks to the Nanostar RTRA project.
1 A.C. Mayer et al., Materials Today 10, 28 (2007).

2 "Organic Photovoltaics: Concepts and Realization" Eds. C. Brabec, V. Dyakonov, J. Parisi, N. S. Sariciftci, Springer Series in Materials Science (2003).

3 M. Reyes-Reyes, K. Kim, D. Carroll, Appl. Phys. Lett. 87, 083506 (2005).

${ }^{4}$ C.J. Brabec et al., Adv. Matt. 22, 3839 (2010).

5 M.C. Scharber et al., Adv. Mater. 18, 789 (2006).

${ }^{6}$ F.B. Kooistra et al., Org. Lett. 9, 551-554 (2007).

7 S. Park et al., Nature Photonics 3, 297 (2009).

8 H-Y. Chen et al., Nature Photonics 3, 649 (2009).

${ }^{9}$ R. Schueppel et al., Phys. Rev. B 77, 085311 (2008).

10 F. Lincker et al., Adv. Func. Mat. 18, 3444 (2008).

11 A.D. Becke, J. Chem. Phys. 98, 1372 (1993).

12 J.P. Perdew, M. Ernzerhof, and K. Burke, J. Chem. Phys. 105, 9982 (1996).

13 L. Hedin, Phys. Rev. 139, A796 (1965).

14 M.S. Hybertsen and S.G. Louie, Phys. Rev. B 34, 5390 (1986).

15 R.W. Godby, M. Schlüter, and L.J. Sham, Phys. Rev. B 37, 10159 (1988).

16 G. Onida, L. Reining, A. Rubio, Rev. Mod. Phys. 74, 601 (2002).

17 W.G. Aulbur, L. Jonsson, J.W. Wilkins, "Quasiparticle calculations in solids", in Solid State Physics: Advances in Research and Applications, Vol.54, 1-218 (2000).

18 A. Szabo and N.S. Ostlund, in "Modern Quantum Chemistry: Introduction to Advanced Electronic Structure Theory", Dover publications (1996).

19 P.C. Martin and J. Schwinger, Phys. Rev. 115, 1342 (1959).

20 N. Dori et al., Phys. Rev. B 73, 195208 (2006).

21 M.L. Tiago, P.R.C. Kent, R.Q. Hood, F.A. Reboredo, J. Chem. Phys. 129, 084311 (2008).

22 M. Palummo et al., J. Chem. Phys. 131, 084102 (2009).

23 P. Umari, G. Stenuit, S. Baroni, Phys. Rev. B 79, 201104 (R) (2009).

24 Y. Ma, M. Rohlfing, C. Molteni, Phys. Rev. B 80, 241405(R) (2009).

25 O. Inganäs et al., Adv. Matter 22, E100 (2010); and references therein.

26 The availability of experimental results for organic molecules in the gas phase is also an important factor directing our choice.

27 X. Blase and P. Ordejón, Phys. Rev. B 69, 085111 (2004).

28 R. Bauernschmitt, Marco Häser, Oliver Treutler, Reinhart Ahlrichs, Chem. Phys. Lett. 264, 573 (1997).

29 Michael Rohlfing, Peter Krüger, and Johannes Pollmann, Phys. Rev. B 52, 1905 (1995).

30 B. Farid, in Electron Correlation in the Solid State, Editor N. H. March, World Scientific Publishing (1999), page 217; and references therein.

31 José M. Soler et al., J. Phys.: Condens. Mater 14, 27452779 (2002).

${ }^{32}$ In good agreement with the results of Ref. 33 in the case of small molecules, we have verified in the case of the polyacenes that a triple- $\zeta+$ double polarization (TZDP) basis for expending the Kohn-Sham orbitals increases the quasiparticle correction to the ionization energy by less than $0.07 \mathrm{eV}$ as compared to the DZP basis adopted in the present study.

33 C. Rostgaard, K.W. Jacobsen, K.S. Thygesen, Phys. Rev. B 81, 085103 (2010).

34 The cutoff radius used by the SIESTA package is chosen large enough so that the "normalization spilling" induced by truncating the gaussian tails is less that $10^{-10}$.

35 M.S. Kaczmarski, Y.C. Ma, M. Rohlfing, Phys. Rev. B 81, 115433 (2010).

${ }^{36}$ F. Bruneval and X. Gonze, Phys. Rev. B 78, 085125 (2008).

37 F. Giustino, M.L. Cohen, S.G. Louie, Phys. Rev. B 81, 115105 (2010).

38 J.A. Berger, L. Reining, F. Sottile, Phys. Rev. B 82, 041103 (2010).

39 D. Foerster and P. Koval, J. Chem. Phys. 131, 044103 (2009).

40 For example, see: A. Ipatov et al., J. Molec. Struct. (Theochem) 762, 179 (2006).

41 C.M. Reeves and M.C. Harrison, J. Chem. Phys. 39, 11 (1963).

42 K. Ruedenberg, R.C. Raffenetti, R.D. Bardo, in Energy, Structure and Reactivity, Proceedings of the 1972 Boulder Conference on Theoretical Chemistry; Wiley: New York (1973).

43 For a recent analysis, see: I. Cherkes, S. Klaiman, N. Miseyev, Int. Jo. Quant. Chem. 109, 2996 (2009).

44 F. Aryasetiawan and O. Gunnarsson, Phys. Rev. B 49, 16214-22 (1994).

45 I.S. Gradshteyn and I.M. Ryzhik, "Tables of integrals, series and products", 7th Edition, Edited by A. Jeffrey and D. Zwillinger, Academic Press (2007).

${ }^{46}$ W.H. Press, S.A. Teukolsky, W.T. Vetterling, B.P. Flannery, in "Numerical Recipes in Fortran", $2^{\text {nd }}$ Edition, Cambridge University Press, p. 252.

47 Experimental data are taken from the NIST chemistry webbook at: http://webbook.nist.gov/chemistry/. The band gap are calculated as the difference of the experimental ionisation energy and electronic affinity. We adopt the most recent experimental data. The provided ionization energies are the vertical ones.

48 J. Berkowitz, J. Chem. Phys 70, 2819 (1979). Previously calculated values using $\triangle \mathrm{SCF}$ and transition state (TS) 
techniques yield $6.52 \mathrm{eV}$ and $6.55 \mathrm{eV}$ respectively. See: M.S. Liao and S. Scheiner, J. Chem. Phys. 114, 9780 (2001).

49 T. Pasinzki, M. Krebsz, G. Vass, J. Mol. Struct. 966, 85 (2009).

50 J.P. Perdew, K. Burke, M. Ernzerhof, Phys. Rev. Lett. 77, 3865 (1996).

51 T. A. Niehaus, M. Rohlfing, F. Della Sala, A. Di Carlo, and Th. Frauenheim, Phys. Rev. A 71, 022508 (2005).

${ }^{52}$ The B3LYP/6-31G(d) HOMO-LUMO gap of $\mathrm{C}_{60}$ was found to be $2.77 \mathrm{eV}$ in Refs. 53 and 54 and that of the $\mathrm{H}_{2} \mathrm{P}$ porphyrin was shown to be $2.9 \mathrm{eV}$ in Ref. 55 .

53 M.K. Shukla and J. Leszczynski, Chem. Phys. Lett. 428, 317 (2006).

54 Z. Zhang et al., J.Phys. Chem. C 112, 19158 (2008).

55 K.A. Nguyen, P.N. Day, and R. Pachter, J. Chem. Phys. 110, 9135 (1999).

56 We observe that our largest discrepancies with planewave based $G_{0} W_{0}(\mathrm{LDA})$ calculations occurs for rather large systems. Further work is needed to know is such discrepancies stem from the gaussian basis or possibly from the difficulty in converging planewave calculations in terms of unit cell and dielectric matrix size or number of conduction bands. Tests on benzene with the PWSCF/Yambo packages 57,58 show that too small a cutoff on the dielectric matrix size, or the summation over a too limited number of conduction bands, open the band gap as a result both of underscreening (unconverged $\mathrm{W}$ ) and by neglecting correlation with higher states (unconverged G).

57 P. Giannozzi et al., J. Phys. Condens. Matter 21, 395502 (2009).

58 A. Marini, C. Hogan, M. Grüning, D. Varsano, Comput. Phys. Commun. 180, 1392 (2009).

59 Apart form the band gap, the opening up to $1.5 \mathrm{eV}$ for the HOMO/HOMO-1 energy difference in PTCDA, (instead of $0.7 \mathrm{eV}$ LDA value), and up to $1.2 \mathrm{eV}$ for the $\mathrm{HOMO} / \mathrm{HOMO}-2$ energy difference in $\mathrm{H}_{2} \mathrm{P}$ (instead of $0.83 \mathrm{eV}$ LDA value) are in excellent agreement with the planewave $G_{0} W_{0}(\mathrm{LDA})$ calculations of Refs. 20 and 22 .

60 P.H. Hahn, W.G. Schmidt and F. Bechstedt, Phys. Rev. B 72, 245425 (2005). 